\title{
Erratum: Dark photons from the center of the Earth: Smoking-gun signals of dark matter [Phys. Rev. D 93, 015014 (2016)]
}

\section{Jonathan L. Feng, Jordan Smolinsky, and Philip Tanedo}

(Received 9 October 2017; published 6 November 2017)

DOI: 10.1103/PhysRevD.96.099901

The code used to produce the final results and plots in this paper contained a typographical error in the dark photon decay length. None of the equations in the paper needs to be modified, but Figs. 3, 4, and 7 should be replaced by the updated versions included here.

In accordance with the corrected results of our analysis, the range of values of $\varepsilon$ accessible to a search for dark photons from the center of the Earth should be $10^{-10} \lesssim \varepsilon \lesssim 10^{-8}$. The mass ranges of dark matter and dark photons accessible to our proposed search are unchanged.

Our paper includes two benchmark points to estimate the number of signal events and describe their kinematic properties. In Sec. IV. A, this benchmark point for IceCube should read, "As a benchmark for IceCube reach, the parameters $m_{X}=1 \mathrm{TeV}, m_{A^{\prime}}=500 \mathrm{MeV}$, and $\varepsilon=5 \times 10^{-9}$ give an expected 40 muon events in ten live years." Similarly,
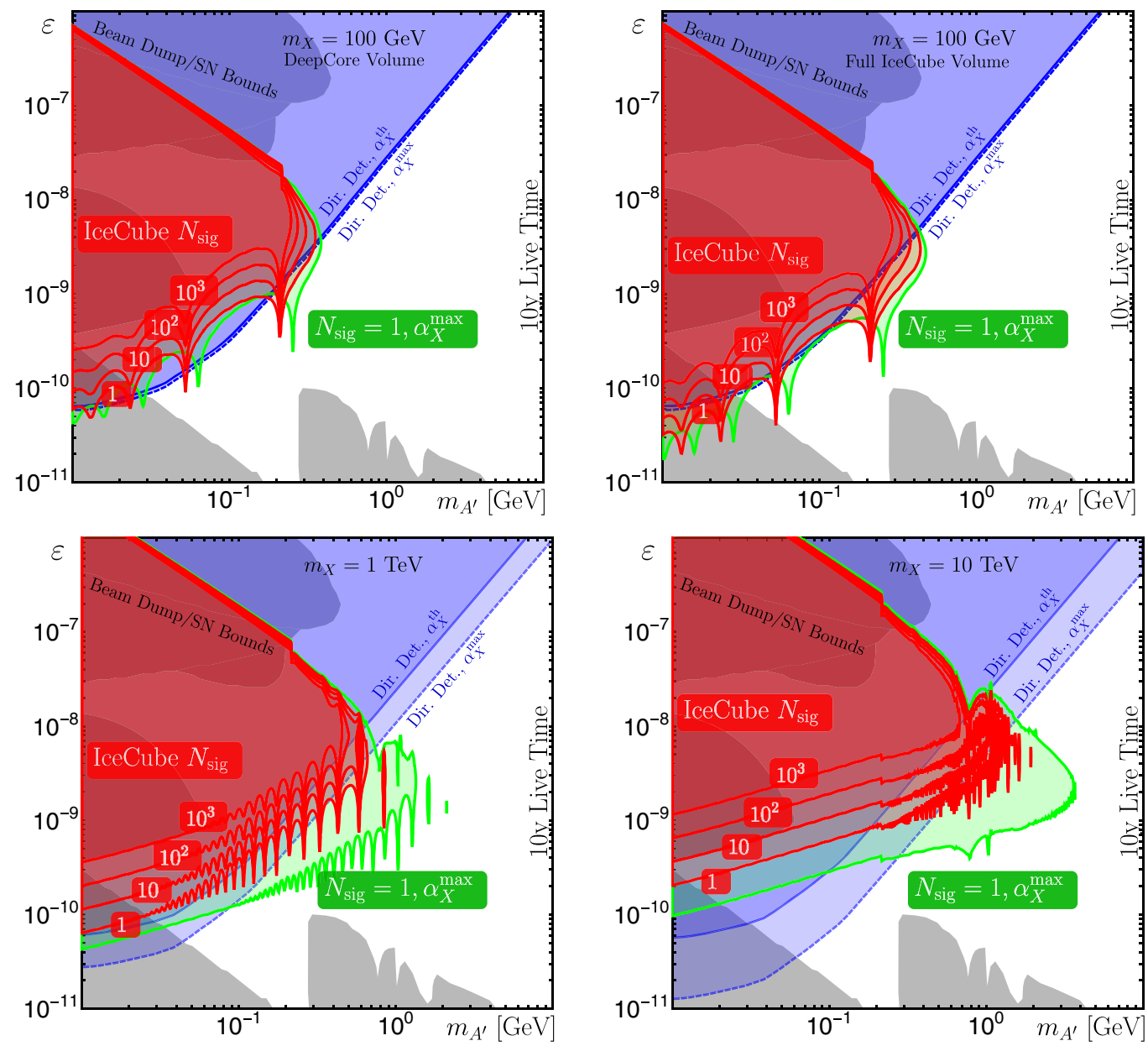

FIG. 3. Red: IceCube event rates for $T=10$ years live time in the $\left(m_{A^{\prime}}, \varepsilon\right)$ plane for $m_{X}=100 \mathrm{GeV}$ in DeepCore (top left) and IceCube (top right), $m_{X}=1 \mathrm{TeV}$ in IceCube (bottom left), and $m_{X}=10 \mathrm{TeV}$ in IceCube (bottom right). The dark sector fine-structure constant is set to the value $\alpha_{X}^{\text {th }}$, which realizes $\Omega_{X} \simeq 0.23$. Green: Single event reach for the maximal dark fine-structure constant $\alpha_{X}^{\max }$ allowed by cosmic microwave background distortion bounds [1]. Blue: current bounds from direct detection [2]. Gray: regions probed by beam dump and supernovae constraints [3-7]. 

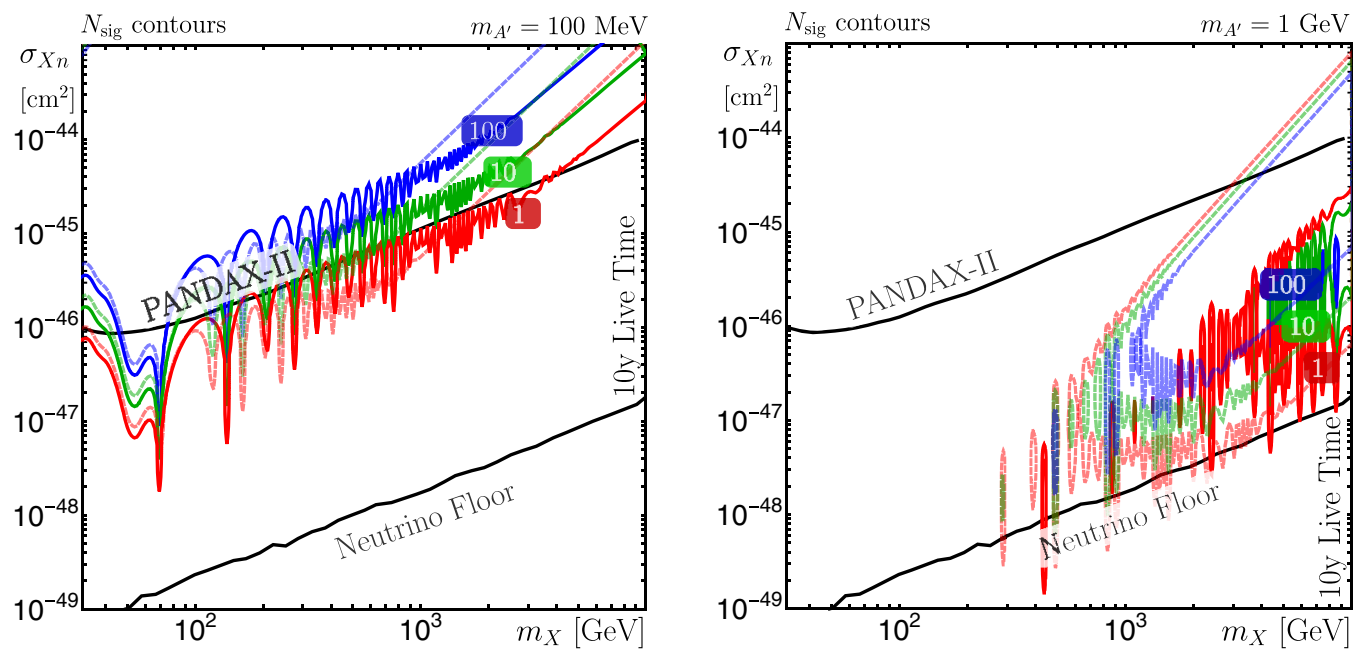

FIG. 4. Comparison of indirect and direct detection sensitivities in the $\left(m_{X}, \sigma_{X n}\right)$ plane for $m_{A^{\prime}}=100 \mathrm{MeV}$ (left) and $1 \mathrm{GeV}$ (right). The direct detection bounds are from the LUX Collaboration [8]. In this regime, the interaction is effectively pointlike in contrast to the low $m_{A^{\prime}}$ region $[2,9,10]$ in Fig. 1, where the direct detection bounds become independent of $m_{A^{\prime}}$ for low $m_{A^{\prime}}$. Also shown is the "neutrino floor," where coherent neutrino scattering affects direct detection experiments [11]; the dashed line is an extrapolation.
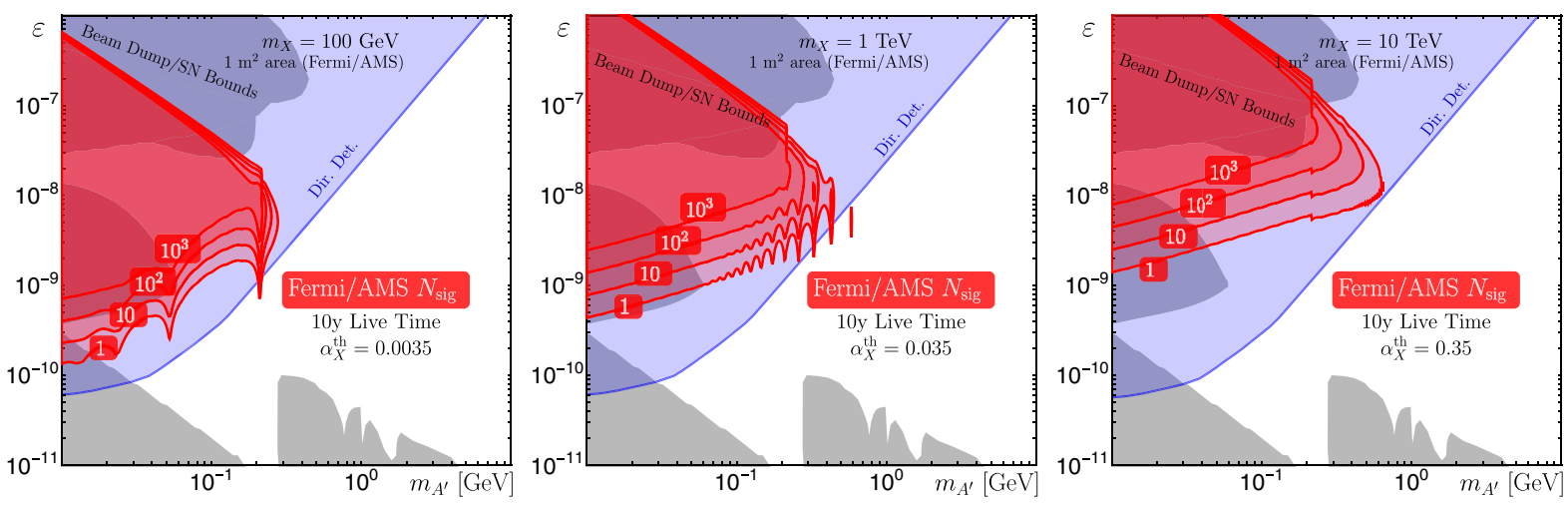

FIG. 7. Ten year signal event rates for a space-based detector in low Earth orbit, such as Fermi/AMS. Colors and bounds are the same as in Fig. 1.

the benchmark point given for Fermi-LAT/AMS-02 in Sec, IV.B should read, "As a benchmark, consider the parameters $m_{X}=1 \mathrm{TeV}, m_{A^{\prime}}=400 \mathrm{MeV}$, and $\varepsilon=10^{-8}$, for which one expects $N_{\text {sig }}=10$ signal events in ten live years."

We are grateful to Adam Green for bringing the error to our attention.

[1] T. R. Slatyer, Indirect dark matter signatures in the cosmic dark ages I. Generalizing the bound on s-wave dark matter annihilation from Planck, Phys. Rev. D 93, 023527 (2016).

[2] E. Del Nobile, M. Kaplinghat, and H.-B. Yu, Direct detection signatures of self-interacting dark matter with a light mediator, J. High Energy Phys. 10 (2015) 055.

[3] J. B. Dent, F. Ferrer, and L.M. Krauss, Constraints on Light Hidden Sector Gauge Bosons from Supernova Cooling, arXiv:1201.2683.

[4] H. K. Dreiner, J.-F. Fortin, C. Hanhart, and L. Ubaldi, Supernova constraints on $\mathrm{MeV}$ dark sectors from $e^{+} e^{-}$annihilations, Phys. Rev. D 89, 105015 (2014).

[5] R. Essig et al., in Community Summer Study 2013: Snowmass on the Mississippi (CSS2013), Minneapolis, MN, 2013, 2013 (unpublished), arXiv:1311.0029.

[6] D. Kazanas, R. N. Mohapatra, S. Nussinov, V.L. Teplitz, and Y. Zhang, Supernova bounds on the dark photon using its electromagnetic decay, Nucl. Phys. B890, 17 (2014). 
[7] E. Rrapaj and S. Reddy, Nucleon-nucleon bremsstrahlung of dark gauge bosons and revised supernova constraints, Phys. Rev. C 94, 045805 (2016).

[8] D. S. Akerib et al. (LUX Collaboration), First Results from the Lux Dark Matter Experiment at the Sanford Underground Research Facility, Phys. Rev. Lett. 112, 091303 (2014).

[9] M. Kaplinghat, S. Tulin, and H.-B. Yu, Direct detection portals for self-interacting dark matter, Phys. Rev. D 89, 035009 (2014).

[10] H. An, M. Pospelov, J. Pradler, and A. Ritz, Direct detection constraints on dark photon dark matter, Phys. Lett. B 747, 331 (2015).

[11] J. Billard, L. Strigari, and E. Figueroa-Feliciano, Implication of neutrino backgrounds on the reach of next generation dark matter direct detection experiments, Phys. Rev. D 89, 023524 (2014). 